hep-th/0303084

LYCEN 2003-02

UFES-DF-OP2003/2

August 21, 2018

\title{
Topological Yang-Mills Theories and Their Observables: a Superspace Approach ${ }^{1}$
}

\author{
José Luis Boldo*, Clisthenis P. Constantinidis****, \\ François Gieres***, Matthieu Lefrançois*** and Olivier Piguet* \\ * Universidade Federal do Espírito Santo (UFES), CCE, Departamento de Física, \\ Campus Universitário de Goiabeiras, BR-29060-900 - Vitória - ES (Brasil) \\ ** The Abdus Salam ICTP, Strada Costiera 11, I - 34014 - Trieste (Italy) \\ *** Institut de Physique Nucléaire, Université Claude Bernard (Lyon 1), \\ 43, boulevard du 11 novembre 1918, F - 69622 - Villeurbanne (France) \\ E-mails: jboldo@cce.ufes.br, clisthen@cce.ufes.br, \\ gieres@ipnl.in2p3.fr, lefrancois@ipnl.in2p3.fr, piguet@cce.ufes.br
}

\begin{abstract}
Witten's observables of topological Yang-Mills theory, defined as classes of an equivariant cohomology, are re-obtained as the BRST cohomology classes of a superspace version of the theory.
\end{abstract}

\section{Introduction}

Observables in topological theories are global, such as knot invariants in ChernSimons theory, Wilson loops, etc. The problem of finding all of them is a problem of

\footnotetext{
${ }^{1}$ Talk presented by Olivier Piguet at the 2nd International Londrina Winter School Mathematical Methods in Physics, 2002, Londrina, Brazil.
} 
"equivariant cohomology", as already pointed out by Witten in 1988 in the framework of 4-dimensional topological Yang-Mills theory [1, 2, 3]. In the latter case, there exists an equivalent approach based on a superspace formalism introduced soon thereafter by Horne 4, 5, which reduces the problem to that of looking for the BRST cohomology in a supersymmetric context. Our aim is to present and develop this formalism, as well as to generalize it to other models, such as topological gravity for instance.

This talk is the summary of a more extended work in preparation $[6]$.

\section{4-Dimensional Topological Yang-Mills Theory}

The gauge connection is given by the matrix valued 1 -form $a=a_{\mu}^{a} T_{a} d x^{\mu}$, where the matrices $T_{a}$ form a basis of the gauge Lie algebra, with

$$
\left[T_{a}, T_{b}\right]=f_{a b}^{c} T_{c}, \quad \operatorname{Tr}\left(T_{a} T_{b}\right)=\delta_{a b}
$$

Topological Yang-Mills theory[1] is characterized, beyond the usual Yang-Mills gauge invariance, by the "shift supersymmetry" defined by the infinitesimal transformations

$$
\tilde{Q} a=\psi, \quad \tilde{Q} \psi=-d \phi-[a, \phi], \quad \tilde{Q} \phi=0,
$$

where the components of the 1-form $\psi$ are fermions and $\phi$ is a bosonic 0-form.

The infinitesimal gauge transformations parametrized by $\omega$ read as

$$
\delta_{\mathrm{YM}} a=d \omega+[a, \omega], \quad \delta_{\mathrm{YM}} \psi=[\psi, \omega], \quad \delta_{\mathrm{YM}} \phi=[\phi, \omega] .
$$

One checks that the square of the supersymmetry operator $\tilde{Q}$ is equal to an infinitesimal gauge transformation, with parameter $\omega$ substituted by the field $\phi$. Thus $\tilde{Q}$ is nilpotent on gauge invariant quantities. This led Witten to define the observables of the theory as the cohomology classes of $\tilde{Q}$ in the space of the gauge invariant operators, which is a problem of equivariant cohomology [2, 3]: a gauge invariant operator $\mathcal{O}$ belongs to the equivariant cohomology of $\tilde{Q}$ if

$$
\tilde{Q} \mathcal{O}=0, \quad \text { but } \quad \mathcal{O} \neq \tilde{Q} \mathcal{P}, \quad \text { with the conditions: } \quad \delta_{\mathrm{YM}} \mathcal{O}=0, \quad \delta_{\mathrm{YM}} \mathcal{P}=0
$$

One can also interpret this formalism as the gauge fixing of the shift supersymmetry considered as a local "gauge" invariance, with a Yang-Mills-invariant gauge fixing condition of the type $f(a) \equiv d a+a^{2}=0$, or $f=* f$, where $*$ is the Hodge operator. Then, $\psi$ is the ghost and $\phi$ its ghost of ghost, $\tilde{Q}$ being the corresponding BRST operator. 


\section{Superspace Formalism}

Enlarging the $d$-dimensional spacetime, of coordinates $x^{\mu}(\mu=0, \cdots, d-1)$, with one fermionic dimension described by a Grassmann (i.e. anticommuting) coordinate $\theta$, with $\theta^{2}=0$, we define a superfield as a superspace function

$$
F(x, \theta)=f(x)+\theta f_{\theta}^{\prime}(x),
$$

which transforms under a supersymmetry (SUSY) transformation as

$$
Q F=\partial_{\theta} F \quad \text { or, in components: } Q f=f^{\prime}, \quad Q f^{\prime}=0 .
$$

By construction the SUSY operator is strictly nilpotent: $Q^{2}=0$. Introducing the differential $d \theta$ - a commuting quantity - we may define $p$-superforms,

$$
\hat{\Omega}_{p}(x, \theta)=\sum_{k=0}^{p} \Omega_{p-k}(x, \theta)(d \theta)^{k},
$$

where $\Omega_{p-k}(x, \theta)=\omega_{p-k}(x)+\theta \omega_{p-k}^{\prime}(x)$ is a $(p-k)$-form in $x$-space with superfield coefficients, as well as the exterior superderivative:

$$
\hat{d}=d+d \theta \partial_{\theta}=d x^{\mu} \partial_{\mu}+d \theta \partial_{\theta}, \quad \text { with } \quad \hat{d}^{2}=0 .
$$

A supergauge theory will be based on a superconnection, the 1-superform ${ }^{2}$

$$
\hat{A}=A(x, \theta)+d \theta A_{\theta}(x, \theta)=a(x)+\theta \psi(x)+d \theta(\chi(x)+\theta \phi(x)),
$$

a Faddeev-Popov ghost 0-superform

$$
C(x, \theta)=c(x)+\theta c^{\prime}(x),
$$

and the corresponding BRST transformations

$$
s \hat{A}=-(\hat{d} C+[\hat{A}, C]) \quad, \quad s C=-C^{2}, \quad s^{2}=0 .
$$

The latter read, in components:

$$
\begin{array}{lll}
s a=-D c, & s \psi=-[c, \psi]-D c^{\prime}, & s \chi=-[c, \chi]-c^{\prime}, \\
s \phi=-[c, \phi]-\left[\chi, c^{\prime}\right], & s c=-c^{2}, & s c^{\prime}=-\left[c, c^{\prime}\right] .
\end{array}
$$

\section{The Wess-Zumino gauge:}

One observes that the component field $\chi(x)$ is a pure gauge degree of freedom w.r.t. to the $c^{\prime}$-transformation in (3.3). The gauge invariance corresponding to the ghost $c^{\prime}(x)$ can be fixed by the "Wess-Zumino gauge" condition $\chi=0$. This condition, which is not preserved by the SUSY transformations (3.1), is however stable with respect to the modified [7] transformations $\tilde{Q}=Q+\left.s\right|_{\chi=0, c=0, c^{\prime}=\phi}$ obtained by adding to the SUSY operator $Q$ a field dependent supergauge transformation. It is easy to check that this new SUSY transformation reproduces, for the "physical" fields $a, \psi$ and $\phi$, the original transformations given by (2.2).

\footnotetext{
${ }^{2}$ All forms and superforms in this paper are Lie algebra valued: $\Phi=\Phi^{a} T_{a}$, see (2.1).
} 


\section{Cohomology of the SUSY operator $Q$ :}

According to (3.1), the component fields are grouped in doublet representations of the nilpotent superspace SUSY operator $Q$. It follows [8, 9] that the cohomology of $Q$ is trivial: every form or superform which is $Q$-invariant is the $Q$-variation of another form or superform. This obviously distinguishes $Q$ from its Wess-Zumino gauge version $\tilde{Q}$ which is nilpotent only when applied to gauge invariant quantities, and moreover has a nontrivial equivariant cohomology [1].

\section{Observables in the superspace formalism}

The equivalence of the superspace theory with the one originally proposed by Witten, the latter being a Wess-Zumino gauge fixed version of the former, suggests to define an observable $\mathcal{O}$ in superspace as a BRST cohomology class:

$$
s \mathcal{O}=0, \quad \text { but } \mathcal{O} \neq s \mathcal{P}
$$

where $\mathcal{O}$ and $\mathcal{P}$ are both $p$-dimensional ${ }^{3}$ space-time integrals:

$$
\mathcal{O}=\int_{M_{p}} \omega_{p}(x), \quad \mathcal{P}=\int_{M_{p}} \phi_{p}(x),
$$

with the conditions:

$$
Q \mathcal{O}=0, \quad Q \mathcal{P}=0
$$

The latter condition and the triviality of the cohomology of $Q$ implies now that, up to a possible total derivative which can be discarded without loss of generality, the integrand of $\mathcal{O}$ - the $p$-form $\omega_{p}$ - is $Q$-exact:

$$
\omega_{p}(x)=Q \Omega_{p}(x, \theta), \quad \text { hence } \mathcal{O}=\int_{M_{p}} Q \Omega_{p}(x, \theta)=\int_{M_{p}} \partial_{\theta} \Omega_{p}(x, \theta),
$$

which is the superspace integral of a "superfield form", i.e. of a space-time form whose coefficients are superfields.

\section{General solution}

The general solution of the BRST cohomology problem (4.1) with the SUSY invariance condition (4.2) consists of the two classes described below. For a proof that there are no other solutions, see Reference [6].

\footnotetext{
${ }^{3}$ The space-time dimension will not be fixed a priori. The space-time integrals are performed over an arbitrary $p$-manifold or submanifold $M_{p}$.
} 


\subsection{Equivariantly Trivial Solutions}

The first class of solutions is of the general form

$$
{ }^{D-p+1} \Delta_{(p)}=\int_{M_{p}} Q^{D-p} \mathcal{H}_{p}\left(F_{A}, \Psi, \Phi, D_{A} \Psi, D_{A} \Phi\right)
$$

where $\mathcal{H}_{p}$ is a gauge invariant function of the superfield forms $F_{A}, \Psi$ and $\Phi$ and their covariant derivatives $D_{A} \Psi=d \Psi+[A, \Psi]$ and $D_{A} \Phi$. These superfield forms are the components of the supercurvature $\hat{F} \equiv \hat{d} \hat{A}+\hat{A}^{2}$,

$$
\hat{F}=F_{A}+\Psi d \theta+\Phi(d \theta)^{2}
$$

with

$$
\begin{aligned}
& F_{A}=d A+A^{2}, \quad \Psi=\partial_{\theta} A+D_{A} A_{\theta}=\psi+D_{a} \chi+O(\theta) \\
& \Phi=\partial_{\theta} A_{\theta}+A_{\theta}^{2}=\phi+\chi^{2}+O(\theta)
\end{aligned}
$$

Their BRST transformations read as

$$
s F_{A}=-\left[C, F_{A}\right], \quad s \Psi=-[C, \Psi], \quad s \Phi=-[C, \Phi] .
$$

The solutions (5.1), although nontrivial in the sense of the BRST cohomology, are trivial in the sense of the equivariant cohomology. Indeed, in the Wess-Zumino gauge, they conserve the same form as in (5.1), but with $A, F_{A}, \Psi$ and $\Phi$ replaced by $a, F_{a}=d a+a^{2}, \psi$ and $\phi$, and the operator $Q$ replaced by $\tilde{Q}$ : the result is then explicitly given by the $\tilde{Q}$-variation of a gauge invariant integral.

\subsection{Equivariantly Nontrivial Solutions}

The solutions of the BRST cohomology problem (4.1) which are nontrivial in the sense of the equivariant cohomology are given in terms of superforms by the following superspace algorithm.

1. Consider all the superforms $\hat{\Omega}_{D}(x, \theta)$ (of ghost number 0 and superform degree $D)$ which are nontrivial solutions of the cohomology $H(s \mid \hat{d})$ of $s$ modulo $\hat{d}$ in the space of the superforms made of polynomials of the basic superforms $\hat{A}(x, \theta)$, $C(x, \theta), \hat{d} \hat{A}(x, \theta)$ and $\hat{d} C(x, \theta)$. Nontriviality in the sense of the cohomology $H(s \mid \hat{d})$ for a superform $\hat{\Omega}$ means

$$
s \hat{\Omega}=0 \quad(\text { modulo } \hat{d}), \quad \text { but } \quad \hat{\Omega} \neq s \hat{\Phi} \quad \text { (modulo } \hat{d}) .
$$

These $\hat{\Omega}_{D}$ are nontrivial solutions of sets of "superdescent equations"

$$
s \hat{\Omega}_{D}+\hat{d} \hat{\Omega}_{D-1}^{1}=0, \quad s \hat{\Omega}_{D-1}^{1}+\hat{d} \hat{\Omega}_{D-2}^{2}=0, \quad \cdots, \quad s \hat{\Omega}_{0}^{D}=0 .
$$


Here the exponent $g$ of the superform $\hat{\Omega}_{p}^{g}$ denotes its ghost number, i.e. its degree in the superfield ghost $C$ or its components. The index $p$ denotes its degree as a superform.

2. Expand $Q \hat{\Omega}_{D}=\partial_{\theta} \hat{\Omega}_{D}$ according to the space-time form degree $p$ :

$$
Q \hat{\Omega}_{D}=\sum_{p=0}^{D} w_{p}(x)(d \theta)^{D-p}
$$

The space-time forms $w_{p}$ are our solutions:

$$
s w_{p}(x)=0 \quad(\text { modulo } d), \quad \text { and } \quad Q w_{p}(x)=0 \text {. }
$$

This follows from the identity $\hat{d} Q F(x, \theta)=d Q F(x, \theta)$, which is a direct consequence of the definition (3.2).

We thus obtain, for a fixed maximum degree $D$, a set of observables

$$
\mathcal{O}=\int_{M_{p}} w_{p}(x)
$$

where the space-time forms $\omega_{p}$ are the coefficients of the superform $Q \hat{\Omega}_{D}$, where $\hat{\Omega}_{D}$ represents a nontrivial solution of the superdescent equations (5.3).

\subsection{Solution of the Superdescent Equation}

The nontrivial observables are thus completely determined from the general solution of the superdescent equations (5.3) . The latter is given by the generalization to the present superspace formalism of standard results of BRST cohomology 9 . The result is:

$$
\hat{\Omega}_{D}=\theta_{r_{1}}^{\mathrm{CS}}(\hat{A}) f_{r_{2}}(\hat{F}) \cdots f_{r_{L}}(\hat{F}), \quad \text { with } \quad D=2 \sum_{i=1}^{L} m_{r_{i}}-1, \quad L \geq 1
$$

where $f_{r}(\hat{F})$ is the supercurvature invariant of degree $m_{r}$ in $\hat{F}$ corresponding to the gauge group Casimir operator of degree $m_{r}$, and $\theta_{r}^{\mathrm{CS}}(\hat{A})$ is the associated ChernSimons form:

$$
\hat{d} \theta_{r}^{\mathrm{CS}}(\hat{A})=f_{r}(\hat{F}) .
$$

We note that the superform degree of the solution (5.4) is odd.

One finally checks that, upon reducing the result to the Wess-Zumino gauge, one recovers the observables originally given by Witten[1]. 


\section{Example}

We consider the case of maximum degree $D=3$. The superdescent equations read as

$$
s \hat{\Omega}_{3}+\hat{d} \hat{\Omega}_{2}^{1}=0, \quad s \hat{\Omega}_{2}^{1}+\hat{d} \hat{\Omega}_{1}^{2}=0, \quad s \hat{\Omega}_{1}^{2}+\hat{d} \hat{\Omega}_{0}^{3}=0, \quad s \hat{\Omega}_{0}^{3}=0 .
$$

The unique nontrivial solution is

$$
\hat{\Omega}_{3}=\operatorname{Tr}\left(\hat{A} \hat{d} \hat{A}+\frac{2}{3} \hat{A}^{3}\right), \quad \hat{\Omega}_{2}^{1}=\operatorname{Tr}(\hat{A} \hat{d} C), \quad \hat{\Omega}_{1}^{2}=\operatorname{Tr}(C \hat{d} C), \quad \hat{\Omega}_{0}^{3}=-\frac{1}{3} \operatorname{Tr} C^{3} .
$$

Note that $\hat{\Omega}_{3}$ is the Chern-Simons superform: $\hat{d} \hat{\Omega}_{3}=\operatorname{Tr}\left(\hat{F}^{2}\right)$.

The observables are then given by the expansion

$$
\partial_{\theta} \hat{\Omega}_{3}=\sum_{p=0}^{3} w_{p}(x)(d \theta)^{3-p},
$$

with

$$
\begin{array}{ll}
w_{0}=\operatorname{Tr}\left(\phi^{2}+2 \phi \chi^{2}\right), & w_{1}=\operatorname{Tr} 2\left(\psi \phi+\phi D \chi+\psi \chi^{2}\right)+d(\cdots) \\
w_{2}=\operatorname{Tr}\left(2 \phi F_{a}+2 \psi D \chi+\psi^{2}\right)+d(\cdots), & w_{3}=\operatorname{Tr}\left(2 \psi F_{a}\right)+d(\cdots)
\end{array}
$$

In the Wess-Zumino gauge $(\chi=0)$ :

$$
\begin{array}{ll}
w_{0}=\operatorname{Tr}\left(\phi^{2}\right), & w_{1}=\operatorname{Tr}(2 \psi \phi)+d(\cdots), \\
w_{2}=\operatorname{Tr}\left(2 \phi F_{a}+\psi^{2}\right)+d(\cdots), & w_{3}=\operatorname{Tr}\left(2 \psi F_{a}\right)+d(\cdots)
\end{array}
$$

which corresponds to Witten's result up to total derivatives.

\section{Conclusion}

The superspace BRST cohomology which we have advocated as an alternative definition of the observables of a topological theory of the Yang-Mills type, reproduces Witten's original result using a rather straightforward extension to superspace of standard results on BRST cohomology [9]. However it is also realized that another type of solutions exists, which are finally proved to be trivial in the sense of equivariant cohomology. The main difficulty which had to be overcome was to show 6 ] that there are no other solutions to the problem of the superspace BRST cohomology. These results lead us to wonder about the applicability of such an approach to the construction of observables in more complex systems, for example topological gravity and Yang-Mills theories with more than one supersymmetry generator. These are problems under current investigation, and we hope to provide answers to these questions soon [10]. 


\section{Acknowledgments}

J.L.B., C.P.C. and O.P. thank the Conselho Nacional de Desenvolvimento Científico e Tecnológico $(\mathrm{CNPq}-$ Brazil) for financial support.

C.P.C. also thanks the Coordenação de Aperfeiçoamento de Pessoal de Nível Superior (CAPES - Brazil) for financial support, and the Abdus Salam International Center for Theoretical Physics (ICTP - Italy) for hospitality during a three months stay under its Associate Program.

O.P. acknowledges the Institut de Physique Nucléaire, Université Claude Bernard (Lyon 1) for its kind hospitality during a one month's stay as Professeur Invité.

\section{References}

[1] E. Witten, Int.J.Mod.Phys. A6 (1991) 2775; Commun.Math.Phys. 117 (1988) 353.

[2] P. van Baal, S. Ouvry and R. Stora, Phys.Lett. B220 (1989) 159.

[3] L. Baulieu and I.M. Singer, Nucl.Phys.(Proc.Suppl.) B5 (1988) 12.

A. Blasi and R. Collina, Phys.Lett. B222 (1989) 419.

R. Stora, F. Thuillier and J. C. Wallet, "Algebraic structure of cohomological field theory models and equivariant cohomology", Lectures at the Caribbean Spring School of Mathematics and Theoretical Physics, St. Francois, Guadeloupe, May 30 - June 13, 1993, preprint ENSLAPP-A-481-94,

F. Delduc, N. Maggiore, O. Piguet and S. Wolf, Phys.Lett. B385 (1996) 132. [hep-th/9605158].

[4] J.H. Horne, Nucl.Phys. B318 (1989) 22.

[5] C. Aragão de Carvalho and L. Baulieu, Phys.Lett. B275 (1992) 323.

[6] J.L. Boldo, C.P. Constantinidis, F. Gieres, M. Lefrançois and O. Piguet, "Observables in topological Yang-Mills theories", work in preparation (now available as: [hep-th/0303053]).

[7] M. Blau and G. Thompson, Commun.Math.Phys. 152 (1993) 41, [hep-th/9112012]; Nucl.Phys. B492 (1997) 545, [hep-th/9612143].

[8] O. Piguet and S.P. Sorella, "Algebraic Renormalization", Lecture Notes in Physics m28, (Springer-Verlag, Berlin 1995).

[9] G. Barnich, F. Brandt and M. Henneaux, Phys.Rep. 338 (2000) 439.

[10] J.L. Boldo, C.P. Constantinidis, F. Gieres, M. Lefrançois, J.A. Nogueira and O. Piguet, work in progress. 